\title{
Localized coherent structures of the Davey-Stewartson equation in the bilinear formalism
}

\author{
L. Martínez Alonso and E. Medina Reus \\ Departamento de Métodos Matemáticos de la Fisica, Facultad de Ciencias Fisicas, Universidad \\ Complutense de Madrid, 28040 Madrid, Spain
}

(Received 4 June 1991; accepted for publication 1 March 1992)

The Davey-Stewartson equation is considered from the point of view of the bilinear formalism of the Kyoto school. Multidromion solutions are constructed in terms of free fermions and their asymptotic properties are characterized. The dynamical properties of dromions are discussed.

\section{INTRODUCTION}

Recent studies on $(2+1)$-dimensional integrable systems have shown the existence of solutions describing localized coherent structures with remarkable dynamical properties. ${ }^{1,2}$ Unlike one-dimensional solitons these structures do not preserve, in general, their form upon interaction $^{2}$ and may undergo processes of fusion and fission. ${ }^{3,4}$ The basic model exhibiting these solutions is the Davey-Stewartson (DS) equation:

$$
\begin{aligned}
& i q_{t}+q_{x x}+q_{y y}+\left(U_{1}+U_{2}\right) q=0, \\
& U_{1 y}=\frac{1}{2}\left(|q|^{2}\right)_{x}, \\
& U_{2 x}=\frac{1}{2}\left(|q|^{2}\right)_{y} .
\end{aligned}
$$

It is important to notice that (1.1) is relevant in many physical situations as it constitutes a generic limit of nonlinear dispersive equations in $2+1$ dimensions. ${ }^{5-7}$ The localized coherent structures of (1.1) are called dromions ${ }^{8}$ and possess a natural spectral interpretation in terms of the inverse scattering transform (IST) method. ${ }^{8-10}$ However, in order to achieve a complete understanding of these novel nonlinear objects as well as to determine the class of integrable systems in which they arise, it is essential to devise alternative methods different from the IST method. In this sense the bilinear approach developed by the Kyoto school ${ }^{11,12}$ constitutes a powerful and elegant formalism which leads to important hierarchies of integrable models and provides a geometrical characterization for relevant classes of solutions.

The present work deals with the bilinear theory of the DS equation and its application to the analysis of the dynamical properties of dromions. Our analysis is based on the formulation of the DS equation as a member of the two-component KP hierarchy. ${ }^{12}$ Throughout the paper the free-fermion language of Refs. 11 and 12 is used which turns out to be particularly useful for characterizing the properties of the dromion interaction. Our main results are the following: (i) A multidromion solution which generalizes the one given in Refs. 2, 8, and 9 is provided. It is characterized in terms of elements of a certain subgroup of $\mathrm{Gl}(2 \infty)$.

(ii) Explicit expressions for the asymptotic trajectories of the dromion maxima in terms of the parameters which determine multidromion solutions are obtained.

(iii) Special types of dromion solutions are studied and conditions are found under which a dromion retains its form upon interaction or vanishes in one of the asymptotic limits $t \rightarrow \pm \infty$.

(iv) The analysis of the dromion interaction shows that contrary to what is claimed in Refs. 2,8 , and 9, the dromion dynamics is not completely determined by the motion of the one-dimensional solitons arising in the boundary functions

$$
u_{1}(t, x)=U_{1}(t, x,-\infty), \quad u_{2}(t, y)=U_{2}(t,-\infty, y)
$$

The paper is organized as follows. Section II is devoted to the description of the free-fermion method of Ref. 12 and its application for solving the two-component KP hierarchy. In Sec. III the DS equation (1.1) is characterized as a bilinear system of the two-component KP hierarchy. It is found that the solutions of the DS equation are associated with a subgroup of the $\mathrm{Gl}(2 \infty)$ invariant under an antilinear operation. We notice that the formulation of the DS equation in terms of Hirota's bilinear equations was already used in Ref. 13 for constructing two-dimensional lump solutions and more recently in Ref. 3 for studying multidromion solutions. The multidromion solutions of the DS equation are introduced in Sec. IV. The corresponding $\tau$ functions are written as vacuum expectation values of certain elements of the Clifford algebra generated by the free fermions. Their explicit expressions are found by using Wick's theorem. Section $\mathrm{V}$ is concerned with the analysis of the dromion dynamical properties. The asymptotic motion of the dromions is characterized by means of explicit formulas for the positions of their maxima. Furthermore, we analyze 
the asymptotic form of the boundary functions (1.2) and study its relationship with the dromion motion. In the last part of Sec. V we describe several interesting types of dynamical situations associated with some special choices of multidromions solutions and discuss the connections of our work with some results of Refs. 2 and 8.

\section{BILINEAR IDENTITIES AND CLIFFORD ALGEBRAS}

The bilinear identities are intimately related to the properties of Clifford algebras. This fact is one of the most interesting aspects of the bilinear formalism and is the origin of an important method of solution for integrable models. ${ }^{11,12}$ In the case of the two-component KP hierarchy the relevant Clifford algebra $C$ is generated by two infinite collections $\psi_{n}^{(i)}, \psi_{n}^{(i) *}(i=1,2 ; n \in \mathrm{Z})$ of anticommuting variables

$$
\left\{\psi_{n}^{(i)}, \psi_{m}^{(j) *}\right\}=\delta^{i j} \delta_{m n}, \quad\left\{\psi_{n}^{(i)}, \psi_{m}^{(j)}\right\}=\left\{\psi_{n}^{(i) *} \psi_{m}^{(j) *}\right\}=0
$$

Natural representations of this structure can be realized in terms of quantum field operators acting on Fock spaces. In our subsequent analysis we will use two Fock spaces $\mathscr{F}$ and $\mathscr{F}$, determined by two vacuum vectors $|\mathrm{vac}\rangle$ and $\langle\mathrm{vac}|$, respectively, satisfying

$$
\begin{array}{ll}
\psi_{n}^{(i)}|\mathrm{vac}\rangle=0 \quad(n<0), \quad \psi_{n}^{(i) *}|\operatorname{vac}\rangle=0 \quad(n \geqslant 0), \\
\langle\operatorname{vac}| \psi_{n}^{(i)}=0 \quad(n \geqslant 0), \quad\langle\operatorname{vac}| \psi_{n}^{(i) *}=0 \quad(n<0) .
\end{array}
$$

Thus we define

$$
\mathscr{F}=\{A|\mathrm{vac}\rangle: A \in C\}, \quad \mathscr{F}=\{\langle\operatorname{vac}| A: A \in C\} .
$$

Some vectors in $\mathscr{F}$ and $\mathscr{F}$ that will play an important role in the next section are given by

$$
\left|l_{2}, l_{1}\right\rangle=A_{l_{2}}^{(2)} A_{l_{1}}^{(1)}|\mathrm{vac}\rangle, \quad\left\langle l_{1}, l_{2}\right|=\langle\operatorname{vac}| B_{l_{1}}^{(1)} B_{l_{2}}^{(2)},
$$

where

$$
\begin{aligned}
& A_{l}^{(i)}= \begin{cases}\psi_{-|l|}^{(i)} \cdots \psi_{-1}^{(i) *} & (l<0), \\
1 & (l=0), \\
\psi_{l-1}^{(i)} \cdots \psi_{0}^{(i)} & (l>0),\end{cases}
\end{aligned}
$$

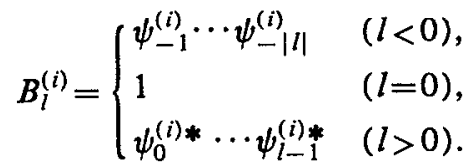

The vacuum expectation value (VEV) of elements $A \in C$ may be defined as

$$
\begin{aligned}
\langle A\rangle & =\langle\operatorname{vac}|A| \operatorname{vac}\rangle \\
& =(\langle\operatorname{vac}|A,| \operatorname{vac}\rangle) \\
& =(\langle\operatorname{vac}|, A| \operatorname{vac}\rangle),
\end{aligned}
$$

where the basic VEV's are those corresponding to quadratic products of generators of $C$. They all vanish with the exception of

$$
\left\langle\psi_{n}^{(i)} \psi_{n}^{(i) *}\right\rangle=\left\langle\psi_{m}^{(i) *} \psi_{m}^{(i)}\right\rangle=1, \quad n<0, \quad m \geqslant 0
$$

The VEV's of more complicated elements of $C$ can be calculated with the help of Wick's theorem.

The relationship between bilinear identities and Clifford algebras lies on an algebraic property satisfied by the following element of $C \otimes C$ :

$$
\Lambda=\sum_{i=1,2} \sum_{n \in Z} \psi_{n}^{(i)} \otimes \psi_{n}^{(i) *}
$$

To describe this property, let us consider the Lie algebra gl $(2 \cdot \infty)$ spanned by the set of quadratic products $\left\{\psi_{n}^{(i)} \psi_{m}^{(j)} *_{: i, j}=1,2 ; n, m \in Z\right\}$ and the unit element $1 \in C$, and let $\mathrm{Gl}(2 \cdot \infty)$ be the corresponding multiplicative group in the Clifford algebra $C$. Then, from the anticommutation relations of the generators of $C$ it follows that ${ }^{12,14}$

$$
\Lambda g \otimes g=g \otimes g \Lambda,
$$

for all $g \in \operatorname{Gl}(2 \cdot \infty)$. As a consequence, it can be shown ${ }^{12}$ that given $g \in \mathrm{Gl}(2 \cdot \infty)$ the functions

$$
\tau_{L M}(z)=\left\langle l_{1}, l_{2}\left|e^{H(z)} g\right| m_{2}, m_{1}\right\rangle,
$$

where $L=\left(l_{1}, l_{2}\right), M=\left(m_{1}, m_{2}\right)$ with $l_{1}+l_{2}=m_{1}+m_{2}$, and

$z=\left(z^{(1)}, z^{(2)}\right) \in \mathbb{C}^{2 \cdot \infty}, H(z)=\sum_{i=1,2} \sum_{n \in Z} z_{n}^{(i)} \sum_{m \in Z} \psi_{m}^{(i)} \psi_{m+n}^{(i) *}$,

satisfy the following identity for a sufficiently large circle $\gamma(|k|=R)$

$$
\begin{aligned}
& \sum_{i=1,2} \int_{\gamma} d k(-1)^{\Sigma_{j>i} l_{j}+l_{j}^{\prime} k^{l_{i}-l_{i}^{\prime}-2} e^{\xi\left(k, z^{(i)}-z^{\left.(i)^{\prime}\right)}\right.}} \\
& \cdot \tau_{L-e_{i} M}\left[z-\epsilon_{i}\left(\frac{1}{k}\right)\right] \tau_{L^{\prime}+e_{r^{\prime}} M^{\prime}}\left[z^{\prime}+\epsilon_{i}\left(\frac{1}{k}\right)\right]=0,
\end{aligned}
$$

where $e_{1}=(1,0), e_{2}=(0,1)$. These kinds of identities are known as bilinear identities.

At this point it is helpful to introduce Hirota's bilinear operators. Given an analytic function $P(z)$ of $z \in \mathbb{C}^{2 \cdot \infty}$, 
the Hirota's operator $P(D) \quad\left[D=\left(D^{(1)}, D^{(2)}\right), \quad D^{(i)}\right.$ $\left.=\left(D_{1}^{(i)}, \ldots, D_{n}^{(i)}, \ldots\right)\right]$ acting on ordered products $\alpha(z) \cdot \beta(z)$ is defined by

$$
P(D) \alpha(z) \cdot \beta(z)=\left.P\left(\partial^{\prime}-\partial^{\prime \prime}\right) \alpha\left(z^{\prime}\right) \cdot \beta\left(z^{\prime \prime}\right)\right|_{z^{\prime}=z^{\prime \prime}=z},
$$

where $\partial=\left(\partial^{(1)}, \partial^{(2)}\right)$ with $\partial^{(i)}=\left(\partial / \partial z_{1}^{(i)}, \ldots, \partial / \partial z_{n}^{(i)}, \ldots\right)$. There are many interesting properties satisfied by these objects. For instance, the following obvious identity

$$
\begin{aligned}
\exp (a D) \alpha(z) \cdot \beta(z)= & \alpha(z+a) \beta(z-a), \\
& a D=\sum_{i=1,2} \sum_{n \in Z} a_{n}^{(i)} D_{n}^{(i)},
\end{aligned}
$$

helps us to analyze the system (2.4). Indeed, by performing the change of variables $z \rightarrow z-u, z^{\prime} \rightarrow z+u$ in (2.4) we get

$$
\begin{aligned}
& \int_{\gamma} d k \sum_{i=1,2} k^{r^{(i)}} e^{\xi\left(k,-2 u^{(i)}\right)} \exp \left\{\left[u+\epsilon_{i}\left(\frac{1}{k}\right)\right] D\right\} \\
& \times \tau_{L^{\prime}+e_{i} M^{\prime}}^{(i)}(z) \cdot \tau_{L-e_{i} M}^{(i)}(z)=0, \quad l=1,2,3,4 .
\end{aligned}
$$

We may evaluate these integrals by means of the residue at $k=\infty$ of their integrands. To this end it is convenient to introduce the elementary Schur polynomials $S_{n}(v)$ depending on $v=\left(v_{1}, \ldots, v_{n}, \ldots\right) \in \mathbb{C}^{\infty}$. They are defined as the coefficients of the expansion

$$
\exp \sum_{n=1}^{\infty} k^{n} v_{n}=\sum_{n=1}^{\infty} k^{n} S_{n}(v) .
$$

From (2.6) one obtains the general expression

$$
S_{n}(v)=\sum_{[\alpha]=n} \frac{1}{\alpha !} v^{\alpha}, \quad n>0 .
$$

Here the sum extends to all $\alpha=\left(\alpha_{1}, \ldots, \alpha_{n}, \ldots\right)$ with integers components $\alpha_{i} \geqslant 0$ such that $[\alpha]=\alpha_{1}+2 \alpha_{2}$ $+\cdots+m \alpha_{m}+\cdots=n$, and

$$
\alpha !=\prod_{n=1}^{\infty}\left(\alpha_{n} !\right), \quad v^{\alpha}=\prod_{n=1}^{\infty} v_{n}^{\alpha_{n}} .
$$

Considering now the coefficient of $k^{-1}$ in the Laurent expansion of the integrands in (2.5) we get

$$
\begin{aligned}
& \frac{1}{\gamma !} P_{\sigma, r_{l}(1)+1}\left(D^{(1)}\right) D^{(2) \gamma} \tau_{L^{\prime}+e_{r} M^{\prime}}^{(1)}(z) \tau_{L-e_{i} M}^{(1)}(z) \\
& \quad+\frac{1}{\sigma !} P_{\gamma, r_{l}^{(2)}+1}\left(D^{(2)}\right) D^{(1) \sigma} \tau_{L^{\prime}+e_{i} M}^{(2)}(z) \tau_{L^{\prime}+e_{i} M}^{(2)}(z)=0,
\end{aligned}
$$

where we denote

$$
P_{\sigma, r}\left(D^{(i)}\right)=\sum_{\alpha+\beta=\sigma} \frac{(-2)^{|\alpha|}}{\alpha ! \beta !} D^{(i) \beta} S_{[\alpha]+r}\left(\widetilde{D}^{(i)}\right),
$$

with $\widetilde{D}^{(i)}=\left(D_{1}^{(i)}, \frac{1}{2} D_{2}^{(i)}, \ldots, \frac{1}{n} D_{n}^{(i)}, \ldots\right)$ and the sum extends to all multi-indices $\sigma=\left(\sigma_{1}, \ldots, \sigma_{n}, \ldots\right), \quad \gamma=\left(\gamma_{1}, \ldots, \gamma_{n}, \ldots\right)$ with $\sigma_{n} \geqslant 0, \gamma_{n} \geqslant 0$. This system of equations is equivalent to the bilinear identity (2.4) and constitutes the hierarchy of bilinear equations associated with the twocomponent KP hierarchy.

\section{THE DAVEY-STEWARTSON EQUATION}

In order to get the DS equation we define the following functions

$$
\begin{aligned}
& \tau_{1}(z)=\left\langle l_{1}, l_{2}-1\left|e^{H(z)} g\right| l_{2}-l-1, l_{1}+l\right\rangle, \\
& \tau_{2}(z)=\left\langle l_{1}+1, l_{2}-2\left|e^{H(z)} g\right| l_{2}-l-1, l_{1}+l\right\rangle, \\
& \tau_{3}(z)=\left\langle l_{1}-1, l_{2}\left|e^{H(z)} g\right| l_{2}-l-1, l_{1}+l\right\rangle,
\end{aligned}
$$

then, the DS equation is related to five members of the system of bilinear equations (2.9), namely,

$$
\begin{aligned}
& D_{2}^{(2)} \tau_{2} \cdot \tau_{1}+D_{1}^{(2)^{2}} \tau_{1} \cdot \tau_{2}=0, \\
& D_{2}^{(1)} \tau_{1} \cdot \tau_{2}+D_{1}^{(1)^{2}} \tau_{2} \cdot \tau_{1}=0, \\
& D_{2}^{(2)} \tau_{1} \cdot \tau_{3}+D_{1}^{(2)^{2}} \tau_{3} \cdot \tau_{1}=0 \\
& D_{2}^{(1)} \tau_{3} \cdot \tau_{1}+D_{1}^{(1)^{2}} \tau_{1} \cdot \tau_{3}=0, \\
& D_{1}^{(1)} D_{1}^{(2)} \tau_{1} \cdot \tau_{1}-2 \tau_{2} \cdot \tau_{3}=0,
\end{aligned}
$$

corresponding, respectively, to the choices

$$
\begin{array}{lll}
l=1, & \sigma=(0, \ldots, 0, \ldots), & \gamma=(0,1,0, \ldots, 0, \ldots) ; \\
l=1, & \sigma=(0,1,0, \ldots, 0, \ldots), & \gamma=(0, \ldots, 0, \ldots) ; \\
l=2, & \sigma=(0, \ldots, 0, \ldots), & \gamma=(0,1,0, \ldots, 0, \ldots) ; \\
l=2, & \sigma=(0,1,0, \ldots, 0, \ldots), & \gamma=(0, \ldots, 0, \ldots) ; \\
l=3, & \sigma=(1,0, \ldots, 0, \ldots), & \gamma=(0, \ldots, 0, \ldots) .
\end{array}
$$

If we introduce the map $z\left(t, x, y, t^{\prime}\right)$ $=\left[z^{(1)}\left(t, x, y, t^{\prime}\right), z^{(2)}\left(t, x, y, t^{\prime}\right)\right]$ from $\mathbb{C}^{4}$ into $\mathbb{C}^{2 \cdot \infty}$ given by

$$
\begin{aligned}
& z_{1}^{(1)}=\frac{x}{2}, z_{2}^{(1)}=-i \frac{t}{4}+i \frac{t^{\prime}}{4}, z_{1}^{(2)}=\frac{y}{2}, z_{2}^{(2)}=i \frac{t}{4}+i \frac{t^{\prime}}{4}, \\
& z_{n}^{(i)}=0, \quad n>2,
\end{aligned}
$$

and we define

$$
p=\tau_{2} / \tau_{1}, \quad q=\tau_{3} / \tau_{1}, \quad U=\log \tau_{1},
$$


then, the bilinear equations (3.2) yield the following system of differential equations:

$$
\begin{aligned}
& -i p_{t}+p_{x x}+p_{y y}+2\left(U_{x x}+U_{y y}\right) p=0 \\
& i q_{t}+q_{x x}+q_{y y}+2\left(U_{x x}+U_{y y}\right) q=0 \\
& q p=4 U_{x y} .
\end{aligned}
$$

Notice that (3.5) does not involve any differentiation with respect to the $t^{\prime}$ variable. Therefore, in what follows we shall set $t^{\prime}=0$ and we shall denote $z(t, x, y) \equiv z(t, x, y, 0)$. Now, if $(t, x, y) \in \mathbb{R}^{3}$ and the reduction condition

$$
\tau_{1}=\tau_{1}^{*}, \quad \tau_{3}=\tau_{2}^{*},
$$

is satisfied, then (3.5) reduces to the system

$$
i q_{t}+q_{x x}+q_{y y}+2\left(U_{x x}+U_{y y}\right) q=0, \quad|q|^{2}=4 U_{x y},
$$

which in turn implies that $q$ satisfies the DS equation

$$
\begin{aligned}
& i q_{t}+q_{x x}+q_{y y}+\left(U_{1}+U_{2}\right) q=0, \\
& U_{1}=\frac{1}{2} \int_{-\infty}^{y} d y^{\prime}|q|_{x}^{2}+u_{1}, \\
& U_{2}=\frac{1}{2} \int_{-\infty}^{x} d x^{\prime}|q|_{y}^{2}+u_{2},
\end{aligned}
$$

where

$$
\begin{aligned}
& u_{1}(t, x)=2 \partial_{x}^{2} \log \tau_{1}(z(t, x,-\infty)), \\
& u_{2}(t, y)=2 \partial_{y}^{2} \log \tau_{1}(z(t,-\infty, y)) .
\end{aligned}
$$

Notice that according to (3.7) and the last equation in (3.4) we have also that

$$
|q|^{2}=4 \partial_{x y} \log \tau_{1}(z(t, x, y))
$$

It is thus obvious that $\tau_{1}$ constitutes the most relevant object in the bilinear theory of the DS equation.

Therefore, (3.1) provides a method for generating solutions of the two-component KP hierarchy of bilinear equations. In what follows the simplest choice $l=l_{1}=l_{2}$ $-1=0$ will be assumed, so that taking into account that $H(z)|\mathrm{vac}\rangle=0$, the functions (3.1) reduce to the VEV's

$$
\begin{aligned}
& \tau_{1}(z)=\langle g(z)\rangle, \quad \tau_{2}(z)=\left\langle\psi_{0}^{(1) *} \psi_{-1}^{(2)} g(z)\right\rangle, \\
& \tau_{3}(z)=\left\langle\psi_{-1}^{(1)} \psi_{0}^{(2) *} g(z)\right\rangle,
\end{aligned}
$$

where

$$
g(z)=e^{H(z)} g e^{-H(z)}, \quad g \in \mathrm{Gl}(2 \cdot \infty) .
$$

Since we are interested in the DS equation, it is essential to know under what conditions on $g \in \operatorname{Gl}(2 \cdot \infty)$ the functions (3.11) satisfy the reduction condition (3.6). This question may be conveniently formulated in terms of the antilinear isomorphism $T: C \rightarrow C$ defined by

$$
\begin{aligned}
& T(1)=1, \quad T\left(\psi_{n}^{(i)}\right)=(-1)^{i+n} \psi_{-n-1}^{(i) *}, \\
& T\left(\psi_{n}^{(i) *}\right)=(-1)^{i+n} \psi_{-n-1}^{(i)} .
\end{aligned}
$$

Observe that the VEV of a quadratic product of the generators of $\mathrm{C}$ remains invariant under the action of $\mathrm{T}$. Moreover, according to Wick's theorem, these VEV's generate the VEV of any element of $C$. Therefore, taking into account the antilinear character of $T$ we deduce

$$
\langle T(A)\rangle=\langle A\rangle^{*}, \quad A \in C .
$$

This property suggests to introduce the group $G_{0}$ $=\{g \in \mathrm{Gl}(2 \infty): T(g)=g\}$. Indeed, for real $(t, x, y)$ it is easy to see that the operator $H(z(t, x, y))$ of $(2.3)$ remains invariant under $T$. As a consequence, given $g \in G_{0}$ then for all $(t, x, y) \in \mathbb{R}^{3}$ the product

$$
g(z(t, x, y))=e^{H(z(t, x, y))} g e^{-H(z(t, x, y))}
$$

belongs to $G_{0}$ too, and therefore (3.13) implies

$$
\begin{aligned}
\tau_{1} & =\langle g(z(t, x, y))\rangle=\langle T[g(z(t, x, y))]\rangle=\langle g(z(t, x, y))\rangle^{*}=\tau_{1}^{*}, \\
\tau_{2} & =\left\langle\psi_{0}^{(1) *} \psi_{-1}^{(2)} g(z(t, x, y))\right\rangle=\left\langle T\left[\psi_{-1}^{(1)} \psi_{0}^{(2) *} g(z(t, x, y))\right]\right\rangle \\
& =\left\langle\psi_{-1}^{(1)}\left[\psi_{0}^{(2) *} g(z(t, x, y))\right]\right\rangle^{*}=\tau_{3}^{*} .
\end{aligned}
$$

In other words, the functions (3.11) determine solutions to the DS equation provided the group element $g \in \mathrm{Gl}(2 \infty)$ is invariant under the antilinear isomorphism $T$ defined by (3.12).

\section{SOLUTIONS OF THE DS EQUATION: COHERENT STRUCTURES}

In the above section we have described a method for generating solutions of the DS equation by means of Clifford algebra. Our next task is to show how the method is used to get an explicit expression for these solutions.

\section{A. Solutions of the DS equation in terms of fermion fields}

Given a circle $\gamma(|k|=R)$ we define the following fermion fields 


$$
\begin{array}{r}
\psi^{(i)}(k)=\sum_{n \in Z} \psi_{n}^{(i)} k^{n}, \psi^{(i) *}(k)=\sum_{n \in Z} \psi_{n}^{(i) *} k^{-n}, \\
i=1,2 ; k \in \gamma,
\end{array}
$$

where $\psi_{n}^{(i)}, \psi_{n}^{(i) *}$ are the generators of the Clifford algebra $C$. They satisfy the anticommutation relations

$$
\begin{aligned}
& \left\{\psi^{(i)}(k), \psi^{(j)}(q)\right\}=\left\{\psi^{(i) *}(k), \psi^{(j) *}(q)\right\}=0, \\
& \left\{\psi^{(i)}(k), \psi^{(j) *}(q)\right\}=\delta^{i j} \delta(k-q), \quad k, q \in \gamma,
\end{aligned}
$$

where $\delta(k-q)$ denotes the delta function on $\gamma$

$\delta(k-q)=\sum_{n \in Z}\left(\frac{k}{q}\right)^{n}, \quad \int_{\gamma} \frac{d k}{2 \pi i k} \delta(k-q) f(k)=f(q)$.

These fermion fields have simple transformation properties under the adjoint action of $\exp H(z)$. Indeed, from (2.3) and (4.1) we may write $H(z)$ as

$$
H(z)=\int_{\gamma} \frac{d k}{2 \pi i k} \sum_{j=1,2} \xi\left(k, z^{(j)}\right) \psi^{(j)}(k) \psi^{(j) *}(k),
$$

so that using (4.2) we deduce

$$
\begin{aligned}
& e^{H(z)} \psi^{(i)}(k) e^{-H(z)}=e^{\xi\left(k, z^{(i)}\right)} \psi^{(i)}(k), \\
& e^{H(z)} \psi^{(i)^{*}}(k) e^{-H(z)}=e^{-\xi\left(k, z^{(i)}\right)} \psi^{(i) *}(k) .
\end{aligned}
$$

In order to construct solutions of the DS equation we require elements of $\mathrm{Gl}(2 \infty)$ which remain invariant under the antilinear isomorphism (3.12). Now, we notice that

$$
\begin{aligned}
& T\left(\psi^{(i)}(k)\right)=(-1)^{i+1}\left(k^{*}\right)^{-1} \psi^{(i) *}\left(-k^{*}\right), \\
& T\left(\psi^{(i) *}(k)\right)=(-1)^{i+1} k^{*} \psi^{(i)}\left(-k^{*}\right) .
\end{aligned}
$$

Consequently, the simplest quadratic expressions in the fermion fields with a well-defined VEV which are invariant under $T$ are

$$
a \psi^{(i)}(k) \psi^{(j) *}\left(-k^{*}\right)+a^{\prime} \psi^{(j)}\left(k^{\prime}\right) \psi^{(i) *}\left(-k^{*}\right),
$$

with $k+k^{\prime *} \neq 0$ and $a k^{\prime *}=(-1)^{i+j} a^{* *} k$. Hence, we may generate solutions of the DS equation by means of elements of $\mathrm{Gl}(2 \infty)$ of the form

$$
g=\exp \sum_{i, j=1,2} \sum_{l=1}^{N^{(i)}} \sum_{m=1}^{N^{(j)}} a_{l m}^{i j} \psi^{(i)}\left(k_{l}^{(i)}\right) \psi^{(j)} *\left(-k_{m}^{(j) *}\right),
$$

where $k_{l}^{(i)}=\left(k_{l}^{(i)}\right)_{R}+i\left(k_{l}^{(i)}\right)_{I}$ are $N^{(i)}$ different points on the circle $\gamma$ such that

$$
k_{l}^{(i)}+k_{l^{\prime}}^{(i) *} \neq 0 ; \quad 1 \leqslant l, l^{\prime} \leqslant N^{(i)}
$$

and the coefficients $a_{l m}^{i j}$ are complex numbers satisfying

$$
a_{l m}^{i j} k_{m}^{(j) *}=(-1)^{i+j}\left(a_{m}^{j i} k_{l}^{(i) *}\right)^{*} .
$$

We are going to see that these group elements determine relevant solutions of the DS equation. To this end, notice that according to (4.4) and taking (3.3) into account we have

$$
g(z(t, x, y))=\exp \sum_{i, j=1,2} \sum_{l=1}^{N^{(i)}} \sum_{m=1}^{N^{(j)}} A_{l m}^{i j}(t, x, y),
$$

where

$$
\begin{aligned}
& A_{l m}^{i j}(t, x, y)= a_{l m}^{i j} \exp \left[\theta_{l}^{(i)}(t, x, y)+\theta_{m}^{(j) *}(t, x, y)\right] \psi^{(i)} \\
& \times\left(k_{l}^{(i)}\right) \psi^{(j) *}\left(-k_{m}^{(j) *}\right), \\
& \theta_{l}^{(1)}=\frac{1}{2} k_{l}^{(1)} x-\frac{i}{4} k_{l}^{(1)^{2}} t, \quad \theta_{l}^{(2)}=\frac{1}{2} k_{l}^{(2)} y+\frac{i}{4} k_{l}^{(2)^{2}} t
\end{aligned}
$$

Furthermore, from (4.2) and (4.6) we deduce that

$$
\left[A_{l m}^{i j}, A_{l^{\prime} m^{\prime}}^{i^{\prime} j^{\prime}}\right]=0, \quad\left(A_{l m}^{i j}\right)^{2}=0, \quad A_{l m}^{i j} A_{l m^{\prime}}^{i j^{\prime}}=A_{l m}^{i j} A_{l^{\prime} m}^{i^{\prime} j}=0 .
$$

Therefore, we may write $(4.8)$ in the form

$$
\begin{aligned}
g(z(t, x, y)) & =\prod_{i, j=1,2} \prod_{l=1}^{N^{(1)}} \prod_{m=1}^{N^{(2)}} \exp A_{l m}^{i j}(t, x, y) \\
& =\prod_{i, j=1,2} \prod_{l=1}^{N^{(1)}} \prod_{m=1}^{N^{(2)}}\left[1+A_{l m}^{i j}(t, x, y)\right],
\end{aligned}
$$

and the terms in the expansion (4.12) may be organized in the form

$$
g(z(t, x, y))=\sum A\left(I_{1}, I_{1}^{*}, I_{2}, I_{2}^{*}\right),
$$

where the sum extends to all the collections of four sets $I_{i}$, $I_{i}^{*} \subset\left\{1, \ldots, N^{(i)}\right\}(i=1,2)$ such that $I_{1} \cup I_{2}$ and $I_{1}^{*} \cup I_{2}^{*}$ have the same number of elements, and the symbol $A\left(I_{1}, I_{1}^{*}, I_{2}, I_{2}^{*}\right)$ denotes the contribution to $g(z(t, x, y))$ of those terms in (4.12) which depended on $(t, x, y)$ according to the exponential function 


$$
\exp \sum_{i=1,2}\left[\sum_{i \in I_{i}} \theta_{l}^{(i)}+\sum_{m \in I_{i}^{*}} \theta_{m}^{(i) *}\right] .
$$

Clearly, we have $A(\phi, \phi, \phi, \phi)=1$. The general term of (4.13) can be written as

$$
A\left(I_{1}, I_{1}^{*}, I_{2}, I_{2}^{*}\right)=\sum \prod_{i, j=1,2} \prod_{l \in I_{i j}} A_{l_{i j}}^{i j}(l)
$$

Here, the sum extends to all the partitions

$$
I_{i}=I_{i 1} \cup I_{i 2}, \quad I_{i 1} \cap I_{i 2}=\phi,
$$

and injective maps $\sigma_{i j}: I_{i j} \rightarrow I_{j}^{*}$, such that

$$
I_{j}^{*}=\sigma_{1 j}\left(I_{1 j}\right) \cup \sigma_{2 j}\left(I_{2 j}\right), \quad \sigma_{1 j}\left(I_{1 j}\right) \cap \sigma_{2 j}\left(I_{2 j}\right)=\phi .
$$

As it was shown in Sec. III, the main object of our analysis is the function $\tau_{1}(z(t, x, y))=\langle g(z(t, x, y))\rangle$, and the only symbols (4.14) which contribute to the function $\tau_{1}$ are those such that $I_{i}$ and $I_{i}^{*}$ have the same number of elements, for $i=1,2$, respectively. Now, we have

$$
\begin{aligned}
\left\langle\psi^{(i)}(k) \psi^{(j)}\left(k^{\prime}\right)\right\rangle & =\left\langle\psi^{(i) *}(k) \psi^{(j) *}\left(k^{\prime}\right)\right\rangle=0, \\
\left\langle\psi^{(i)}(k) \psi^{(j) *}\left(k^{\prime}\right)\right\rangle & =-\left\langle\psi^{(j) *}\left(k^{\prime}\right) \psi^{(i)}(k)\right\rangle \\
& =-\delta^{i j} \sum_{n>0}\left(\frac{k}{k^{\prime}}\right)^{n}=\delta^{i j} \frac{k^{\prime}}{k-k^{\prime}},
\end{aligned}
$$

where the last equality must be understood in the sense of distributions on the circle $\gamma$, acting on functions which vanish in some neighborhood of $k^{\prime}$. Then, with the help of Wick's theorem, the VEV's arising in the expression of $\tau_{1}$ can be explicitly evaluated. For example, given two subsets $I_{i} \subset\left\{1, \ldots, N^{(i)}\right\}(i=1,2)$, from (4.14) it follows that

$$
\begin{aligned}
& \left\langle A\left(I_{1}, I_{1}, I_{2}, I_{2}\right)\right\rangle \\
& \quad=c\left(I_{1}\right) c\left(I_{2}\right) a\left(I_{1}, I_{2}\right) \exp \sum_{i=1,2} \sum_{l \in I_{i}}\left(\theta_{l}^{(i)}+\theta_{l}^{(i) *}\right),
\end{aligned}
$$

where

$$
\begin{aligned}
c\left(I_{i}\right) & =\operatorname{det}\left(\left\langle\psi^{(i)}\left(k_{l}^{(i)}\right) \psi^{(i) *}\left(-k_{m}^{(i) *}\right)\right\rangle\right) \\
& =\prod_{l} \frac{-k_{l}^{(i) *}}{k_{l}^{(i)}+k_{l}^{(i) *}} \prod_{l<m}\left|\frac{k_{l}^{(i)}-k_{m}^{(i)}}{k_{l}^{(i)}+k_{m}^{(i) *}}\right|^{2}, l, m \in I_{i}
\end{aligned}
$$

and

$$
a\left(I_{1}, I_{2}\right)=\sum \epsilon\left(I_{i j}, \sigma_{i j}\right) \prod_{i, j=1,2} \prod_{l \in I_{i j}} a_{l \sigma_{i j}(l)}^{i j}
$$

Here the sum is understood in the same sense as in (4.14) with $I_{i}^{*}=I_{i}$, and $\epsilon\left(I_{i j}, \sigma_{i j}\right)= \pm 1$ is the signature of the permutation of anticommuting variables

$$
\prod_{i=1,2} \prod_{l \in I_{i}} \alpha_{l}^{(i)} \beta_{l}^{(i)} \rightarrow \prod_{i=1,2} \prod_{l \in I_{i}} \alpha_{l}^{(i)} \beta_{\sigma_{i j}^{(i)}(l)}^{(i)}
$$

where $\alpha_{l}^{(i)}=\psi^{(i)}\left(k_{l}^{(i)}\right), \beta_{l}^{(i)}=\psi^{(i) *}\left(-k_{l}^{(i) *}\right)$.

The fermion fields (4.1) are assumed to be defined on a fixed circle $\gamma(|k|=R)$ and, consequently, the solutions of the DS equation associated with the group elements (4.5) - (4.7) depend on the $N^{(1)}+N^{(2)}$ complex variables $k_{l}^{(i)}$ on $\gamma$. However, it is clear that these solutions can be analytically extended to all complex values of $k_{l}^{(i)}$ provided condition (4.6) holds.

\section{B. Coherent structures: The dromion}

Let us consider the simplest case $N^{(1)}=N^{(2)}=1$ for the group element (4.5)-(4.7). The only relevant terms in (4.12) for calculating $\left\{\tau_{i}\right\}_{i=1}^{3}$ are

$$
1+A_{11}^{11}+A_{11}^{22}+A_{11}^{12}+A_{11}^{21}+A_{11}^{12} A_{11}^{21}+A_{11}^{11} A_{11}^{22} .
$$

Thus one finds

$$
\begin{aligned}
& \tau_{1}=1+d_{1} e^{2 \mu_{R} x(t)}+d_{2} e^{2 \lambda_{R} y(t)}+d_{3} e^{2 \mu_{R} x(t)+2 \lambda_{R} y(t)}, \\
& \tau_{3}=\tau_{2}^{*} \\
&=-2 \mu a_{11}^{21} \exp \left[\mu x(t)+\lambda y(t)+i\left(|\mu|^{2}+|\lambda|^{2}\right) t\right], \\
& \mu=\mu_{R}+i \mu_{I}=\frac{1}{2} k_{1}^{(1) *}, \quad \lambda=\lambda_{R}+i \lambda_{I}=\frac{1}{2} k_{1}^{(2)}, \\
& d_{i}=-\frac{a_{11}^{i i} k_{1}^{(i) *}}{2\left(k_{1}^{(i)}\right)_{R}} \quad(i=1,2),
\end{aligned}
$$

$$
\begin{aligned}
& d_{3}=\frac{k_{1}^{(1) *} k_{1}^{(2) *}}{4\left(k_{1}^{(1)}\right)_{R}\left(k_{1}^{(2)}\right)_{R}}\left(a_{11}^{11} a_{11}^{22}-a_{11}^{12} a_{11}^{21}\right), \\
& x(t)=x-2 \mu_{I} t, \quad y(t)=y-2 \lambda_{I} t .
\end{aligned}
$$

For generic positive values of the parameters $d_{i}$ ( $i$ $=1,2,3$ ), the function $q=\tau_{3} / \tau_{1}$ is the dromion solution ${ }^{2}$ of the DS equation. Indeed, to get the expression given in Refs. 2 and 8 observe that (4.20) implies 


$$
|q|=\frac{4|\rho|\left(\left|\mu_{R} \lambda_{R}\right|\right)^{1 / 2} \exp \left[-\mu_{R}\left(x(t)-\xi^{(1)}\right)-\lambda_{R}\left(y(t)-\xi^{(2)}\right)\right]}{\left\{1+\exp \left[-2 \mu_{R}\left(x(t)-\xi^{(1)}\right)\right]\right\}\left\{1+\exp \left[-2 \lambda_{R}\left(y(t)-\xi^{(2)}\right)\right]\right\}+|\rho|^{2}},
$$

where

$$
\rho=\left(\frac{d_{3}}{d_{1} d_{2}}-1\right)^{1 / 2}
$$

and

$$
\xi^{(1)}=-\frac{1}{2 \mu_{R}} \log d_{1}, \quad \xi^{(2)}=-\frac{1}{2 \lambda_{R}} \log d_{2}
$$

It is clear that the dromion represents a perturbation exponentially decaying in all the directions of the $(x, y)$ plane which propagates without deformation. Observe that (4.21) reaches its maximum value at the point $\left(x^{(1)}(t), x^{(2)}(t)\right)=\left(x^{(1)}+2 \mu_{I} t, x^{(2)}+2 \lambda_{I} t\right)$, where

$$
x^{(1)}=\frac{1}{4 \mu_{R}} \log \left(\frac{d_{2}}{d_{1} d_{3}}\right), \quad x^{(2)}=\frac{1}{4 \lambda_{R}} \log \left(\frac{d_{1}}{d_{2} d_{3}}\right) .
$$

These two parameters are the natural ones for characterizing the dromion position and they will be used in our subsequent analysis of the asymptotic properties of multidromion solutions.

An important constant of the motion for the DS equation is

$$
M=\frac{1}{2} \int_{\mathbf{R}^{2}}|q|^{2} d x d y .
$$

This functional is referred to as the energy of the solution $q$. However, we prefer to call it the mass of $q$ since this is its interpretation in the context of Galilean invariant Schrödinger equations. ${ }^{15}$ In the case of the dromion solution (4.20) one finds at once

$$
M=2 \int_{\mathbf{R}^{2}} \partial_{x y} \log \tau_{1} d x d y=2 \log \frac{d_{3}}{d_{1} d_{2}} .
$$

Moreover, from (4.21) and (4.23) it follows that the maximum amplitude of the dromion is

$$
|q|_{\max }=2\left(\lambda_{R} \mu_{R}\right)^{1 / 2}\left|\frac{1-e^{M / 4}}{1+e^{M / 4}}\right|^{1 / 2} .
$$

Other types of coherent structures arise provided one, or several, of the parameters $d_{i}$ in (4.20) vanish. Consider, for example, the following cases $d_{1}=0$ or $d_{2}=0$, $d_{1}=d_{2}=0$. If $d_{1}=0$, the function $|q|$ takes the form of a kink located on the line

$$
\lambda_{R} x(t)+\mu_{R} y(t)=-\log d_{3}^{1 / 2} .
$$

A similar structure results by assuming $d_{2}=0$, while for the choice $d_{1}=d_{2}=0$ the function $|q|$ acquires the form of a moving wall located on the line (4.27). On the other hand, in the case

$$
d_{3}=d_{1} d_{2},
$$

(4.25) implies $M=0$, so that the solution $q$ vanishes identically.

\section{DROMION DYNAMICS}

Once we have seen how the dromion solution of the DS equation arises in the bilinear formalism, we will deal now with the dynamical properties of dromions. Our strategy is based on the asymptotic analysis of the expression

$$
|q(t, x, y)|^{2}=4 \partial_{x y} \log \langle g(z(t, x, y))\rangle,
$$

associated with a group element of the form (4.5)-(4.7). From (4.10) and (4.13) it is clear that the asymptotic behavior of (5.1) is determined by the functions $\left|\exp \theta_{l}^{(i)}\right|$. In this sense, notice that

$$
\begin{aligned}
& \operatorname{Re} \theta_{l}^{(1)}(t, x)=\frac{1}{2}\left(k_{l}^{(1)}\right)_{R}\left(x-v_{l}^{(1)} t\right), \\
& \operatorname{Re} \theta_{m}^{(2)}(t, y)=\frac{1}{2}\left(k_{m}^{(2)}\right)_{R}\left(y-v_{m}^{(2)} t\right),
\end{aligned}
$$

where

$$
\begin{gathered}
v_{l}^{(1)}=-\left(k_{l}^{(1)}\right)_{l}, v_{m}^{(2)}=\left(k_{m}^{(2)}\right)_{l}, \\
l=1, \ldots, N^{(1)}, m=1, \ldots, N^{(2)} .
\end{gathered}
$$

Thus there are $N^{(1)}$ times $N^{(2)}$ characteristic velocities

$$
v_{l m}=\left(v_{l}^{(1)}, v_{m}^{(2)}\right),
$$

associated with (5.1). In what follows, we will restrict ourselves to the analysis of solutions satisfying

$$
\left(k_{l}^{(i)}\right)_{R}>0 \quad l=1, \ldots, N^{(i)}, \quad i=1,2,
$$

and we will assume that the complex numbers $k_{l}^{(i)}$ are ordered so that

$$
v_{1}^{(i)}>v_{2}^{(i)}>\cdots>v_{N^{(i)}}^{(i)}, \quad i=1,2 .
$$




\section{A. Asymptotic motion of dromions}

Consider the limits $t \rightarrow \pm \infty$ of (5.1) on a ray in the $(x, y)$ plane given by

$$
x(t)=x+v_{l_{1}}^{(1)} t, \quad y(t)=y+v_{l_{2}}^{(2)} t
$$

From the form of $\theta_{l}^{(i)}$ one deduces that the dominant terms in the expansion (4.13) of $g(t, x(t), y(t))$ are

$$
\begin{aligned}
& A\left(I_{l_{1}}^{ \pm}, I_{l_{1}}^{ \pm}, I_{l_{2}}^{ \pm}, I_{l_{2}}^{ \pm}\right)+A\left(I_{l_{1}}^{ \pm} \cup\left\{l_{1}\right\}, I_{l_{1}}^{ \pm} \cup\left\{l_{1}\right\}, I_{l_{2}}^{ \pm}, I_{l_{2}}^{ \pm}\right) \\
& \quad+A\left(I_{l_{1}}^{ \pm}, I_{l_{1}}^{ \pm}, I_{l_{2}}^{ \pm} \cup\left\{l_{2}\right\}, I_{l_{2}}^{ \pm} \cup\left\{l_{2}\right\}\right) \\
& \quad+A\left(I_{l_{1}}^{ \pm} \cup\left\{l_{1}\right\}, I_{l_{1}}^{ \pm} \cup\left\{l_{1}\right\}, I_{l_{2}}^{ \pm} \cup\left\{l_{2}\right\}, I_{l_{2}}^{ \pm} \cup\left\{l_{2}\right\}\right),
\end{aligned}
$$

where

$$
I_{l_{i}}^{ \pm}=\left\{1 \leqslant l \leqslant N^{(i)}:+\left(l-l_{i}\right)>0\right\}
$$

Using (4.17)-(4.19) and (5.1) it is found that as $t \rightarrow \pm \infty$, the function $|q(t, x(t), y(t))|$ reduces to the expression corresponding to a function $\tau_{1}$ of the form (4.20), with parameters $\mu=\frac{1}{2} k_{l_{1}}^{(1) *}, \lambda=\frac{1}{2} k_{l_{2}}^{(2)}$ and

$d_{1}^{ \pm}=c_{1}^{ \pm} \frac{a\left(I_{l_{1}}^{ \pm} \cup\left\{l_{1}\right\}, I_{l_{2}}^{ \pm}\right)}{a\left(I_{l_{1}}^{ \pm}, I_{l_{2}}^{ \pm}\right)}, \quad d_{2}^{ \pm}=c_{1}^{ \pm} \frac{a\left(I_{l_{1}}^{ \pm}, I_{l_{2}}^{ \pm} \cup\left\{l_{2}\right\}\right)}{a\left(I_{l_{1}}^{ \pm}, I_{l_{2}}^{ \pm}\right)}$

$d_{3}^{ \pm}=c_{1}^{ \pm} c_{2}^{ \pm} \frac{a\left(I_{l_{1}}^{ \pm} \cup\left\{l_{1}\right\}, I_{l_{2}}^{ \pm} \cup\left\{l_{2}\right\}\right)}{a\left(I_{l_{1}}^{ \pm}, I_{l_{2}}^{ \pm}\right)}$,

where

$$
c_{i}^{ \pm}=\frac{c\left(I_{l_{i}}^{ \pm} \cup\left\{l_{i}\right\}\right)}{c\left(I_{l_{i}}^{ \pm}\right)}, \quad i=1,2 .
$$

Unless otherwise stated we will henceforth assume that the real parameters (5.7) are strictly positive and that $d_{3}^{ \pm} \neq d_{1}^{ \pm} d_{2}^{ \pm}$. Therefore, for each pair of indices $\left(l_{1}, l_{2}\right)$ $\left(1 \leqslant l_{i} \leqslant N^{(i)}\right)$ there is an associated dromion moving with velocity $v_{l_{1} l_{2}}$ in the solution $q(t, x, y)$ of the DS equation. A similar analysis shows that $q(t, x, y)$ vanishes asymptotically on all the rays $x(t)=x+v^{(1)} t, y(t)=y+v^{(2)} t$ for velocities $\left(v^{(1)}, v^{(2)}\right)$ different from (5.2). This means that the solution of the DS equation determined by the group element (4.5)-(4.7) reduces asymptotically to $N^{(1)}$ times $N^{(2)}$ dromions. Henceforth, this solution will be referred to as the $\left(N^{(1)}, N^{(2)}\right)$-dromion solution and the dromion corresponding to each pair $\left(l_{1}, l_{2}\right)$ will be called the $\left(l_{1}, l_{2}\right)$ th dromion. Unlike one-dimensional solitons, in the general case dromions change their form as a result of the interaction proccess. To prove this fact it is enough to observe that as a consequence of (4.25) and (5.7) the mass of the $\left(l_{1}, l_{2}\right)$ th dromion in the limits $t \rightarrow \pm \infty$ is

$$
M_{l_{1} l_{2}}^{ \pm}=2 \log \frac{a\left(I_{l_{1}}^{ \pm} \cup\left\{l_{1}\right\}, I_{l_{2}}^{ \pm} \cup\left\{l_{2}\right\}\right) a\left(I_{l_{1}}^{ \pm}, I_{l_{2}}^{ \pm}\right)}{a\left(I_{l_{1}}^{ \pm} \cup\left\{l_{1}\right\}, I_{l_{2}}^{ \pm}\right) a\left(I_{l_{1}}^{ \pm}, I_{l_{2}}^{ \pm} \cup\left\{l_{2}\right\}\right)},
$$

and, in general, $M_{l_{1} l_{2}}^{+} \neq M_{l_{1} l_{2}}^{-}$.

From (4.23) and (5.7) we may determine the asymptotic trajectories $x^{(i) \pm}(t)$ of the dromions. Thus for the $\left(l_{1}, l_{2}\right)$ th dromion we obtain $x^{(i) \pm}(t)=x_{l_{1} l_{2}}^{(i) \pm}+v_{l_{i}}^{(i)} t$ where $x_{l_{1} l_{2}}^{(i) \pm}$ can be written as a sum of three terms

$$
x_{l_{1} l_{2}}^{(i) \pm}=-\frac{2}{\left(k_{l_{i}}^{(i)}\right)_{R}}\left(\delta_{l_{i}}^{(i) \pm}+\Delta_{l_{1} l_{2}}^{(i) \pm}+\frac{1}{8} M_{l_{1} l_{2}}^{ \pm}\right), \quad i=1,2,
$$

where

$$
\begin{aligned}
& \delta_{l_{i}}^{(i) \pm}=\sum_{l_{i} I_{l_{i}}^{ \pm}} \log \left|\frac{k_{l}^{(i)}-k_{l_{i}}^{(i)}}{k_{l}^{(i)}+k_{l_{i}}^{(i) *}}\right|, \\
& \Delta_{l_{1} l_{2}}^{(1) \pm}=\frac{1}{2} \log \left|\frac{k_{l_{1}}^{(1) *} a\left(I_{l_{1}}^{ \pm} \cup\left\{l_{1}\right\}, I_{l_{2}}^{ \pm}\right)}{2\left(k_{l_{1}}^{(1)}\right)_{R} a\left(I_{l_{1}}^{ \pm}, I_{l_{2}}^{ \pm}\right)}\right|, \\
& \Delta_{l_{1} l_{2} \pm}^{(2) \pm}=\frac{1}{2} \log \left|\frac{k_{l_{2}}^{(2) *} a\left(I_{l_{1}}^{ \pm}, I_{l_{2}}^{ \pm} \cup\left\{l_{2}\right\}\right)}{2\left(k_{l_{2}}^{(2)}\right)_{R} a\left(I_{l_{1}}^{ \pm}, I_{l_{2}}^{ \pm}\right)}\right| .
\end{aligned}
$$

\section{B. Asymptotic motion of the boundary functions}

The dynamical law associated with the DS equation is determined by two types of interaction terms in the differential equation (3.8). First, there is a nonlinear and nonlocal term given by the integrals involved in the sum $U_{1}+U_{2}$ and, second, there is a linear term whose coefficient is the sum $u_{1}+u_{2}$, of the boundary data. We are going now to study the asymptotic forms of the functions $u_{i}$ and their relationship with the dromion motion.

Our starting point is again the group element (4.5)(4.7). In view of (3.9) and (3.11) we may write

$$
\begin{aligned}
& u_{1}(t, x)=2 \partial_{x}^{2} \log \langle g(z(t, x,-\infty))\rangle, \\
& u_{2}(t, y)=2 \partial_{y}^{2} \log \langle g(z(t,-\infty, y))\rangle .
\end{aligned}
$$

Since we are assuming $\left(k_{l}^{(i)}\right)_{R}>0\left(i=1,2 ; l=1, \ldots, N^{(i)}\right)$, from (4.13) it follows that

$$
\begin{aligned}
& g(t, x,-\infty)=\sum A\left(I_{1}, I_{1}^{*}, \phi, \phi\right), \\
& g(t,-\infty, y)=\sum A\left(\phi, \phi, I_{2}, I_{2}^{*}\right),
\end{aligned}
$$


where the sums extend to all pairs of sets $I_{i}, I_{i}^{*}$ $\subset\left\{1, \ldots, N^{(i)}\right\}$ such that $I_{i}$ and $I_{i}^{*}$ have the same number of elements for $i=1,2$, respectively, and $\phi$ denotes the empty set.

The asymptotic analysis of the functions (5.11) can be performed by following the same strategy as in the study of the dromion motion. Thus one proves that as $t \rightarrow \pm \infty$ the boundary function $u_{i}$ decomposes into $N^{(i)}$ solitons which move freely on the trajectories $x^{(i)}(t)$ $=x_{l_{i}}^{(i) \pm}+v_{l_{i}}^{(i)} t$, where $x^{(i) \pm}$ can be written as

$$
x_{l_{i}}^{(i) \pm}=-\frac{2}{\left(k_{l_{i}}^{(i)}\right)_{R}}\left(\delta_{l_{i}}^{(i) \pm}+\Delta_{l_{i}}^{(i) \pm}\right), \quad i=1,2 .
$$

Here $\delta_{l_{i}}^{(i) \pm}$ are given by (5.10a) and

$$
\begin{aligned}
& \Delta_{l_{1}}^{(1) \pm}=\frac{1}{2} \log \left|\frac{k_{l_{1}}^{(1)^{*}} a\left(I_{l_{1}}^{ \pm} \cup\left\{l_{1}\right\}, \phi\right)}{2\left(k_{l_{1}}^{(1)}\right)_{R} a\left(I_{l_{1}}^{ \pm}, \phi\right)}\right|, \\
& \Delta_{l_{2}}^{(2) \pm}=\frac{1}{2} \log \left|\frac{k_{l_{2}}^{(2) *} a\left(\phi, I_{l_{2}}^{ \pm} \cup\left\{l_{2}\right\}\right)}{2\left(k_{l_{2}}^{(2)}\right)_{R} a\left(\phi, I_{l_{2}}^{ \pm}\right)}\right| .
\end{aligned}
$$

Therefore, as $t \rightarrow \pm \infty$ the asymptotic form of the function $u_{1}+u_{2}$ is a traveling rectangular network on the $(x, y)$ plane with $N^{(1)}$ times $N^{(2)}$ nodes which move with the velocities (5.2). Notice that (5.9) and (5.13) have a different structure. In particular, (5.9) shows that the positions of the dromion maxima depend on the parameters of the type $a_{l m}^{i j}$ with $i \neq j$. Then, because of (4.7), it follows that $x_{l_{1} l_{2}}^{(i)}$ depends on both sets of parameters $\left\{k_{l}^{(1)}: l \in I_{l_{1}}^{ \pm} \cup\left\{l_{1}\right\}\right\}$ and $\left\{k_{m}^{(2)}: m \in I_{m_{2}}^{ \pm} \cup\left\{l_{2}\right\}\right\}$. Therefore, the dromion scattering is a two-dimensional dynamical process. In contrast, (5.14) implies that $x_{l_{i}}^{(i) \pm}$ only depends on the parameters $a_{l l}^{i i}$ and $k_{l}^{(i)}$ with $l, l^{\prime} \in I_{l_{i}}^{ \pm}$ $\cup\left\{l_{i}\right\}$. Hence, the scattering of the nodes of $u_{1}+u_{2}$ is essentially one dimensional.

\section{Special types of dromion solutions}

We have discussed so far the properties of the $\left(N^{(1)}, N^{(2)}\right)$-dromion solution of the DS equation for general values of the parameters $k l^{(i)}, a_{l m}^{i j}$ satisfying (4.6) and (4.7) such that $d_{i}^{ \pm}>0(i=1,2,3)$ and $d_{3}^{ \pm} \neq d_{1}^{ \pm} d_{2}^{ \pm}$for all the asymptotic dromions. There are, however, significant departures from the generic behavior of this solution when some special choices for its defining parameters are assumed. We are going to see that dromions may degenerate into kinks or walls, and that processes of fusion and fission of dromions may take place.

In view of the expressions (5.7) for the dromion parameters, it is clear that the symbols $a\left(I_{1}, I_{2}\right)$ defined in (4.19) are of great importance for characterizing the dromion dynamics. Fortunately, there are some particular conditions on the parameters $a_{l m}^{i j}$ which lead to simplifying relations for the symbols $a\left(I_{1}, I_{2}\right)$ involved in (5.7). For example, let us suppose given two subsets $I_{j} \subset\left\{1, \ldots, N^{(j)}\right\} \quad(j=1,2)$ and some integer $1 \leqslant l \leqslant N^{(1)}$, with $l \notin I_{1}$, such that

$$
a_{l l^{\prime}}^{1 j}=0, \quad \forall l^{\prime} \in I_{j}, \quad j=1,2 .
$$

Then, from (4.19) it follows at once that

$$
a\left(I_{1} \cup\{l\}, I_{2}\right)=a_{l l}^{11} a\left(I_{1}, I_{2}\right) .
$$

Analogously, given an integer $1 \leqslant m \leqslant N^{(2)}$, with $m \notin I_{2}$, such that

$$
a_{m m^{\prime}}^{2 j}=0, \quad \forall m^{\prime} \in I_{j}, \quad j=1,2,
$$

we have

$$
a\left(I_{1}, I_{2} \cup\{m\}\right)=a_{m m}^{22} a\left(I_{1}, I_{2}\right) .
$$

Furthermore, given two integers $1 \leqslant l \leqslant N^{(1)}, 1 \leqslant m \leqslant N^{(2)}$, with $l \notin I_{1}, m \notin I_{2}$, satisfying (5.15) and (5.17), from (4.19) one easily deduces that

$$
a\left(I_{1} \cup\{l\}, I_{2} \cup\{m\}\right)=\left(a_{l l}^{11} a_{m m}^{22}-a_{l m}^{12} a_{m l}^{21}\right) a\left(I_{1}, I_{2}\right) .
$$

Let us turn now to the $\left(N^{(1)}, N^{(2)}\right)$-dromion solution generated by the group element (4.5)-(4.7), and assume that there are two integers $\left(l_{1}, l_{2}\right), 1 \leqslant l_{i} \leqslant N^{(i)}$, satisfying (5.15) and (5.17), respectively, with respect to the subsets $I_{l_{i}}^{+}(i=1,2)$ defined in (5.6). In other words,

$$
a_{l_{l_{j}^{\prime}}^{\prime}}^{i j}=0, \quad \forall i, j=1,2, \quad 1 \leqslant l_{j}^{\prime} \leqslant N^{(j)}, \quad l_{j}<l_{j}^{\prime} .
$$

Then, according to (5.7), (5.16), (5.18), and (5.19), as $t \rightarrow+\infty$ the expression for the parameters of the $\left(l_{1}, l_{2}\right)$ th dromion reduce to

$d_{i}^{+}=c_{i}^{+} a_{l_{l_{i}},}^{i i}, \quad(i=1,2) ; d_{3}^{+}=c_{1}^{+} c_{2}^{+}\left(a_{l_{1} l_{1}}^{11} a_{l_{2} l_{2}}^{22}-a_{l_{1} l_{2}}^{12} a_{l_{2} l_{1}}^{21}\right)$.

Similary, if $\left(l_{1}, l_{2}\right)$ satisfy

$$
a_{l_{j}^{\prime}}^{i j}=0, \quad \forall i, j=1,2, \quad 1 \leqslant l_{j}^{\prime} \leqslant N^{(j)}, \quad l_{j}>l_{j}^{\prime} .
$$

Then

$d_{i}^{-}=c_{i}^{-} a_{l_{l} l i}^{i i},(i=1,2), \quad d_{3}^{-}=c_{1}^{-} c_{2}^{-}\left(a_{l_{1} l_{1}}^{11} a_{l_{2} l_{2}}^{22}-a_{i_{1} l_{2}}^{12} a_{l_{2} l_{1}}^{21}\right)$.

Suppose that the condition (5.20) [respectively (5.22)] for $\left(l_{1}, l_{2}\right)$ holds. Then the following particularly interesting solution may be characterized: If $a_{l_{1} l_{2}}^{12}=0$, then $d_{3}^{+}=d_{1}^{+} d_{2}^{+}\left(d_{3}^{-}=d_{1}^{-} d_{2}^{-}\right)$, so that the solution $q$ vanishes 
asymptotically on the ray (5.5) as $t \rightarrow+\infty(t \rightarrow-\infty)$ and the $\left(l_{1}, l_{2}\right)$ th dromion does not arise in that limit. This means that we can generate a solution of the DS equation in which the number of dromions is different at $t \rightarrow+\infty$ and $t \rightarrow-\infty$. These kinds of solutions describe processes of fusion and fission of dromions. ${ }^{4}$ It is worth noticing that according to (5.11) and (5.12) the potentials $u_{1}$ and $u_{2}$ are characterized by the parameters $a_{l m}^{i j}$ involved in the operators of the type $A\left(I_{1}, I_{1}^{*}, \phi, \phi\right)$ and $A\left(\phi, \phi, I_{2}, I_{2}^{*}\right)$, respectively, where $\phi$ denotes the empty set. Now, from (4.9) and (4.14) it is obvious that $A\left(I_{1}, I_{1}^{*}, \phi, \phi\right)$ and $A\left(\phi, \phi, I_{2}, I_{2}^{*}\right)$ depend only on the parameters $a_{l m}^{11}$ and $a_{l m}^{22}$, respectively. In other words, the functions $u_{i}$ do not depend on the remaining parameters $a_{l m}^{12}, a_{l m}^{21}$. Therefore, the condition $a_{l_{1} l_{2}}^{12}=0$ is independent of the form of $u_{i}$ and, nevertheless, it implies the vanishing of the $\left(l_{1}, l_{2}\right)$ dromion in one of the asymptotic limits $t \rightarrow \pm \infty$. This fact together with the differences between the asymptotic motion of the dromion and the potential maxima discussed at the end of Sec. V B, shows that the dromion dynamics is not completely determined by the boundary functions $u_{i}$. Indeed, the potentials $\boldsymbol{u}_{i}$ are determined by the parameters $a_{l m}^{11}$ and $a_{l m}^{22}$ only, while the dromion dynamics is characterized by the whole set $a_{l m}^{i j}(i, j=1,2)$.

If both conditions (5.20) and (5.22) hold, then the parameters of the $\left(l_{1}, l_{2}\right)$ th dromion at $t \rightarrow \pm \infty$ are given by (5.21) and (5.23), respectively, and one finds at once that this dromion has the same form in both limits. Notice, for instance, that (4.25), (5.21), and (5.23) yield

$$
M_{l_{1} l_{2}}^{+}=M_{l_{1} l_{2}}^{-}=2 \log \frac{a_{l_{1} l_{1}}^{11} a_{l_{2} l_{2}}^{22}-a_{l_{1} l_{2}}^{12} a_{l_{2} l_{1}}^{21}}{a_{l_{1} l_{1}}^{11} a_{l_{2} l_{2}}^{22}}
$$

In this case, there are also important simplifications for the expressions (5.9), (5.10), and (5.14). Thus one obtains

$$
\Delta_{l_{1} l_{2}}^{(i) \pm}=\Delta_{l_{i}}^{(i) \pm}=\frac{1}{2} \log \left|\frac{k_{l_{i}}^{(i) *} a_{l_{l_{i}}}^{i i}}{2\left(k_{l_{i}}^{(i)}\right)_{R}}\right|, \quad i=1,2 .
$$

As a consequence of (5.24) and (5.25), it follows that

$$
x_{l_{i}}^{(i) \pm}=x_{l_{1} l_{2}}^{(i) \pm}+\frac{1}{4\left(k_{l_{i}}^{(i)}\right)_{R}} M_{l_{1} l_{2}},
$$

where $M_{l_{1} l_{2}}$ is the dromion mass (5.24). Hence, the corresponding position shifts reduce to

$$
\begin{aligned}
x_{l_{1} l_{2}}^{(i)+}-x_{l_{1} l_{2}}^{(i)-} & =x_{l_{i}}^{(i)+}-x_{l_{i}}^{(i)-} \\
& =-\frac{2}{\left(k_{l_{i}}^{(i)}\right)_{R}}\left(\delta_{l_{i}}^{(i)+}-\delta_{l_{i}}^{(i)-}\right) .
\end{aligned}
$$

Therefore, in this case the dynamics of both the $\left(l_{1}, l_{2}\right)$ th dromion and the $\left(l_{1}, l_{2}\right)$ th node of $u_{1}+u_{2}$ are essentially the same.

Another interesting case, which combines several of the situations considered above takes place when

$$
a_{l m}^{i j}=0, \quad l \neq m, \quad 1 \leqslant l \leqslant N^{(i)}, \quad 1 \leqslant m \leqslant N^{(j)} .
$$

This condition implies that (5.20) and (5.22) are satisfied for all pairs $\left(l_{1}, l_{2}\right)$. Furthermore, since $a_{l_{1} l_{2}}^{12}=0$ for $l_{1} \neq l_{2}$, as $t \rightarrow \pm \infty$ there are no dromions with velocitics $v_{l_{1} l_{2}}$ for $l_{1} \neq l_{2}$. Consequently, the corresponding solution of the DS equation gives rise to $\min \left(N^{(1)}, N^{(2)}\right)$ dromions, which move with velocities $v_{l l}, 1 \leqslant l \leqslant \min \left(N^{(1)}, N^{(2)}\right)$ and preserve their form after interaction.

Finally, it is important to relate our work with the results of Refs. 2 and 8 . The $\left(N^{(1)}, N^{(2)}\right)$-dromion solution introduced in this paper depends on the complex parameters $\left(k_{l}^{(i)}, a_{l m}^{i j}\right), \quad 1 \leqslant l \leqslant N^{(i)}, \quad 1 \leqslant m \leqslant N^{(j)} \quad(i, j=1,2)$ subject to the constraints (4.7). It means that the number of independent parameters is

$$
\begin{aligned}
& 2\left(N^{(1)}+N^{(2)}\right)+N^{(1)} N^{(2)}+\frac{1}{2}\left[N^{(1)}\left(N^{(1)}+1\right)\right. \\
& \left.+N^{(2)}\left(N^{(2)}+1\right)\right] .
\end{aligned}
$$

On the other hand, the $(N, M)$-dromion solution of Ref. 8 contains only

$$
2\left(N^{(1)}+N^{(2)}\right)+N^{(1)} N^{(2)},
$$

independent parameters [in the notation of Ref. 8 they are given by $\left.\left(\mu_{l}, \lambda_{m}, c \tilde{\boldsymbol{c}}_{m}, \rho_{l m}\right), 1 \leqslant l \leqslant N^{(1)}, 1 \leqslant m \leqslant N^{(2)}\right]$. In fact, this latter solution is obtained from ours by setting

$$
a_{l l^{\prime}}^{11}=a_{m m^{\prime}}^{22}=0, \quad l \neq l^{\prime}, \quad m \neq m^{\prime} .
$$

From (5.29) it is easy to recover the results of Ref. 8 . Thus in view of (5.16) and (5.18), we have that (5.29) implies

$$
\begin{aligned}
& a\left(I_{l_{1}}^{ \pm} \cup\left\{l_{1}\right\}, \phi\right)=a_{l_{1} l_{1}}^{11} a\left(I_{l_{1}}^{ \pm}, \phi\right), \\
& a\left(\phi, I_{l_{2}}^{ \pm} \cup\left\{l_{2}\right\}\right)=a_{l_{2} l_{2}}^{22} a\left(\phi, I_{l_{2}}^{ \pm}\right),
\end{aligned}
$$

for all $1 \leqslant l_{i} \leqslant N^{(i)}(i=1,2)$, so that $(5.14)$ reads

$$
\Delta_{l_{i}}^{(i) \pm}=\frac{1}{2} \log \left|\frac{k_{l_{i}}^{(i) *} a_{l_{l_{i}}}^{i i}}{2\left(k_{l_{i}}^{(i)}\right)_{R}}\right|
$$

Then (5.13) for the parameters $x_{l_{i}}^{(i) \pm}$ which describe the dynamics of the boundary functions $u_{i}$ reduces to the expression obtained in Ref. 8. Note, in particular, that 


$$
x_{l_{i}}^{(i)+}-x_{l_{i}}^{(i)-}=-\frac{2}{\left(k_{l_{i}}^{(i)}\right)_{R}}\left(\delta_{l_{i}}^{(i)+}-\delta_{l_{i}}^{(i)-}\right)
$$

In Ref. 8 the parameters $x_{l_{i}}^{(i) \pm}$ are used to characterize the dromion motion of the DS equation. Nevertheless, as it is shown by (5.9) and (5.13), they differ from the dromion-maxima positions $x_{l_{1} l_{2}}^{(i)}$ in an essential way. This is particularly obvious for the case considered in Ref. 8. Indeed, if (5.29) holds then from (5.9) and (5.32) we have

$$
\begin{aligned}
x_{l_{1} l_{2}}^{(i)}-x_{l_{1} l_{2}}^{(i)-}= & \left(x_{l_{i}}^{(i)+}-x_{l_{i}}^{(i)-}\right)-\frac{2}{\left(k_{l_{i}}^{(l)}\right)_{R}}\left[\left(\Delta_{l_{1} l_{2}}^{(i)}+-\Delta_{l_{1} l_{2}}^{(i)-}\right)\right. \\
& \left.+\frac{1}{8}\left(M_{l_{1} l_{2}}^{+}-M_{l_{1} l_{2}}^{-}\right)\right] .
\end{aligned}
$$

Observe that the position shifts of the dromion maxima depend on the change of amplitude suffered by the dromions through the mass-increment term in (5.33). However, this dynamical effect is not exhibited by the position shift (5.32).

\section{ACKNOWLEDGMENTS}

The authors would like to thank Professor M. Jaulent for many helpful conversations and the Laboratoire de Physique-Mathematique of Montpellier for kind hopitality while part of this work was done. Financial support from the Dirección General de Investigación Científica y Técnica DGICYT is also aknowledged.

${ }^{1}$ M. Boiti, J. Leon, L. Martina, and F. Pempinelli, Phys. Lett. A 132, 432 (1988).

${ }^{2}$ A. S. Fokas and P. M. Santini, Phys. Rev. Lett. 63, 1329 (1989).

${ }^{3} J$. Hietarinta and R. Hirota, Phys. Lett. A 145, 237 (1990); see also M. Jaulent and L. Martínez Alonso, "Multidimensional solitons in terms of Fermions," in Inverse Problems in Action, edited by P. C. Sabatier (Springer-Verlag, Berlin, 1990).

${ }^{4}$ R. Hernández Heredero, L. Martínez Alonso, and E. Medina Reus, Phys. Lett. A 152, 37 (1991); M. Jaulent, M. A. Manna, and L. Martínez Alonso, Phys. Lett. A 151, 303 (1990).

${ }^{5}$ A. Davey and K. Stewartson, Proc. R. Soc. London, Ser. A 338, 101 (1974).

${ }^{6} \mathrm{M}$. J. Ablowitz and H. Segur, Solitons and the Inverse Scattering Transform (SIAM, Philadelphia, 1981).

${ }^{7}$ F. Calogero and W. Eckhaus, Inv. Probl. 3, L27 (1987).

${ }^{8}$ P. M. Santini, Physica D 41, 26 (1990).

${ }_{9}^{9}$ A. S. Fokas and P. M. Santini, Physica D 44, 99 (1990).

${ }^{10} \mathrm{M}$. Boiti, J. Leon, and F. Pempinelli, "On the Spectral Theory for the Davey-Stewartson Equation," in Inverse Problems in Action, edited by P. C. Sabatier (Springer-Verlag, Berlin, 1990).

${ }^{11}$ E. Date, M. Kashiwara, and T. Miwa, "Transformation Groups for Soliton Equations," in Nonlinear Integrable Systems-Classical Theory and Quantum Theory, edited by M. Jimbo and T. Miwa (World Scientific, Singapore, 1983).

${ }^{12}$ M. Jimbo and T. Miwa, Publ. RIMS Kyoto Univ. 19, 943 (1983).

${ }^{13}$ J. Satsuma and M. J. Ablowitz, J. Math. Phys. 20, 1496 (1979).

${ }^{14} \mathrm{~V}$. G. Kac and D. H. Peterson, "Lectures on the Infinite Wedge Representation and the MKP Hierarchy," Sem. Math. Sup. Vol. 102, (Presse Univ. Montreal, Montreal 1986).

${ }^{15} \mathrm{~J}$. M. Levy-Leblond, "Galilei Group and Galilean Invariance" in Group Theory and its Applications II, edited by E. M. Loebl (Academic, New York, 1971). 
Journal of Mathematical Physics is copyrighted by the American Institute of Physics (AIP). Redistribution of journal material is subject to the AIP online journal license and/or AIP copyright. For more information, see http://ojps.aip.org/jmp/jmpcr.jsp Copyright of Journal of Mathematical Physics is the property of American Institute of Physics and its content may not be copied or emailed to multiple sites or posted to a listserv without the copyright holder's express written permission. However, users may print, download, or email articles for individual use. 